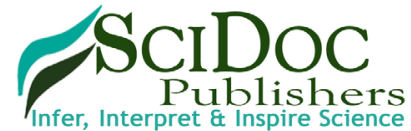

International Journal of Surgery and Research (IJSR)

ISSN 2379-156X

\title{
Effects of Smoking on Hand Tendon Repair: Scientific Study \& Literature Review
}

Review Article

Samona J*, Samona S, Gilin M, Carwile D, Persons S, AG Dass

McLaren Regional Medical Center, Orthopaedic Surgery Department, S. Ballenger Highway, Flint, MI, USA.

Abstract

Purpose: The clinical effect of smoking on after tendon repair in smokers vs non-smokers was investigated, via exploring effects on flexor and tendon repair, and incidence of wound complications.

Methods: Retrospective review of patients treated by 1 orthopedic surgeon, fellowship trained in hand surgery, and 3 occupational therapists working together in 1 physical therapy institution. 56 patients (20 smokers and 36 non-smokers) with acute traumatic tendon lacerations, fixed via direct (end-to-end) method, within 3 weeks from date of injury. Total Active Motion (TAM) was measured via American Society for Surgery of the Hand protocol.

Results: Overall percentage of TAM regained in smokers was $70 \%$ vs $75 \%$ of TAM by non-smokers. No significant differences in TAM between smokers and non-smokers. Flexor tendon TAM was significantly higher in both the smoking and non-smoking groups vs extensors tendon TAM, displaying a larger effect of smoking on flexor vs extensor tendons. No significant dose dependent effect in "heavy" vs "light" smokers on numerous parameters. When grouping excellent, good and fair results, vs poor results, no significant difference exists, telling us tendon repairs universally do "poor" in terms of TAM, despite the fact if they are flexor or tendon repairs, and if the patient is a smoker or not. "Surgical wound complications", were exceptionally low, 1 in the smokers, 2 in the non-smokers.

Conclusion: Despite the undisputable harsh effects of smoking, it is scientifically inaccurate to claim patient post-operative range of motion in flexor and extensor tendon injuries is significantly affected by this unhealthy habit. Nor can we claim surgical site complications are more common in smokers in the setting of such injuries.

Study/Level Of Evidence: (Level 3) Retrospective chart review \& Review of the Literature. Therapeutic \& Prognostic Analysis.

\section{Introduction}

Cigarette smoking has numerous well documented systemic sequela in the medical literature. The Center for Disease Control reports $20.6 \%$ of all adults are regular smokers $(23.5 \%$ of men vs $17.9 \%$ of women), correlating toover 46 million U.S. adults [1]. Male smokers lose an average of 13.2 years of their life, vs 14.5 years for females. Smoking has been proven through multiple studies to be deleterious to the healing process and tendon health. Associations have been established between smoking and rotator cuff tendon repair, shoulder pain, distal bicep tendon rupture, and reduced achilles tendon healing [2-5]. A positive correlation exists between average number of cigarettes smoked and rotator cuff tear presence and size [3]. Smokers have a 7.5 times greater risk of distal biceps tendon rupture vs non-smokers [4]. Nicotine is responsible for delayed tendon-to-bone healing [6], as well as inferior biomechanical properties of native tendons in smokers and tendon grafts used in reconstruction efforts. The biomechanical effects of smoking have a clinically significant effect on post-operative patients in terms of inferior functional outcomes, increased pain [7], increased surgical site infection rates $[8,9]$, skin necrosis, flap failure and wound complications $[6,7$, $10,11]$.

Effects of smoking on tendon repairs of the hand are less understood. Justan et al., proposes smoking increases range of motion (ROM) with 2 stage flexor tendon reconstructions [12]. On the contrary, Trumble et al., claims decreased ROM for zone two flexor injuries in smokers undergoing surgical repair [13]. Both these papers contain inconsistencies and confounding variables which skew the overall results. In previous studies of hand tendon injuries, only flexor tendon injuries have been investigated, extensors were excluded from the studies. The effect of smoking on ROM with acute direct repairs (within 3 weeks

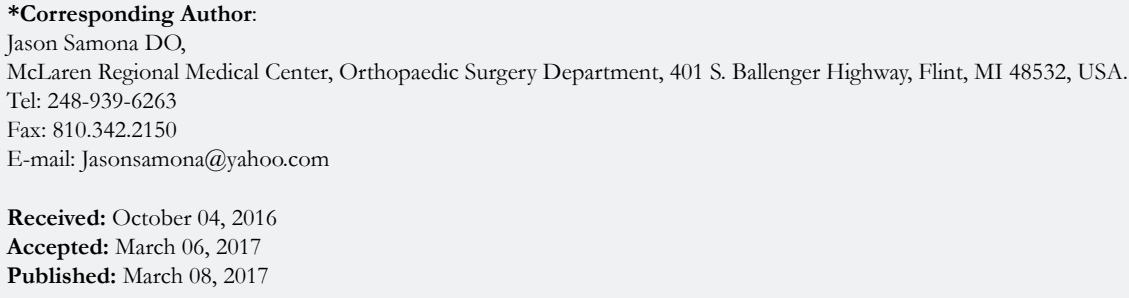

Copyright: Samona J ${ }^{\circ}$ 2017. This is an open-access article distributed under the terms of the Creative Commons Attribution License, which permits unrestricted use, distribution and reproduction in any medium, provided the original author and source are credited. 
of injury) of traumatic tendon injuries (flexor and extensor) has never been investigated. In addition, no studies have been completed regarding rate of surgical wound complications with hand tendon repairs.

The main focus of the study was the clinical effect of smoking on digit ROM, and therefore functional capabilities after tendon repair. The authors anticipated decreased total active motion (TAM) in patients whom smoke. The anecdotal experience of the senior author with over 20 years of experience treating such tendon injuries, in smokers and non-smokers, is which lead this hypothesis. The incidence of wound complications in the patient population was investigated in regards to smokers and non-smokers. It was speculated the smoking group would have a statistically significant higher rate of wound complications. The authors believed both flexor tendons and extensor tendons will be equally affected by the biological effects of smoking.

\section{Materials and Methods}

This was an IRB approved retrospective chart review of patients treated by 1 orthopedic surgeon, fellowship trained in hand surgery, and 3 occupational therapists working together in the same institution. The same surgical methodology and suture type was used in every case. A direct end-to-end repair via Ethicon suture (Ethicon US, LLC) was used to fix all tendon repairs. A modified Kessler technique was used to repair flexor tendon injuries via 3.0 ethibond, and 5.0 nylon used for epitendinous repair. 4.0 ethibond was used in the repair of flexor tendon injuries of the small finger. 3.0 ethibond was used in treatment of extensor tendon injuries, in a figure-of-eightmanner. An identical occupational therapy regimen was used for all patients. These measures were taken to maintain consistency with surgical repair and rehabilitation received, in order to eliminate confounding variables.

Sample size was chosen based on; availability of patient records and those whom completed the standard occupational therapy protocol. Inclusion criteria included; $18-75$ years of age, tendon laceration secondary to acute trauma with acute repair (within 3 weeks of initial injury), zone 2 flexor tendon injuries or digit extensor tendon injuries distal to the juncture tendinae. Exclusion criteria included; patients on chemotherapeutic agents, use of a graft in the repair, long term steroids, immune modulator therapy, alcoholics, malnourished, neurovascular injury to the effected extremity, crush injuries, polytrauma to the digit in question, polytrauama elsewhere at the time of injury, adjacent digits with tendon injuries, ipsilateral upper extremity injury effecting ROM, history of neurological or musculoskeletal disorder or previous injury which limited the patient's pre-injury range of hand motion. Patients with both flexor and extensor tendon injuries on the same hand were excluded from the study as well. The study involved 56 patients ( 20 smokers and 36 non-smokers). Of the smokers, 9 were extensor tendon injuries and 11 were flexor tendon injuries. Of the non-smokers, 15 were extensor tendon injures and 21 were flexor tendons.

TAM was measured in a standard manner by all 3 occupational therapists. Standard accepted values of "normal" for digits 2-4 (240 degrees) and the thumb (260 degrees) were used in the study [14-16]. The patient's TAM was calculated in a standard manner, as set forth by the (ASSH) American Society for Surgery of the Hand. The Strickland method and ASSH-TAM measurements were used by Justan, whereas Trumble only used the measurements of the PIP and DIP in a manner similar but not identical to the Strickland method for ROM analysis (Table 1). For thumb TAM in relation to the ASSH-TAM equation, the thumb IP joint correlated to the DIP joint, the MP joint correlated to the PIP joint, and the Carpal-metacarpal joint correlated to the MP joint in the equation, therefore allowing us to maintain consistency in the calculations. At the cessation of rehabilitation, TAM was measured and compared to the normal values, and a percentage of normal was derived. As set forth by the ASSH, this percentage was then classified as "excellent", "Good", "Fair", or "Poor" based on the percentage of TAM regained (Table 2).

Medical records of these patients were searched for "surgical wound complications" in the initial 6 months post operatively.

Table 1. Formula for the American Society for Surgery of the Hand measure of (TAM) Total Active Motion.

FORMULA FOR THE AMERICAN SOCIETY FOR SURGERY OF THE HAND MEASURE OF (TAM) TOTAL ACTIVE MOTION;

Degrees TAM $=($ MP flexion + PIP flexion + DIP flexion $)-($ MP extension deficit + PIP extension deficit + DIP extension deficit)

$\mathrm{AM}=$ Total Active Motion

$\mathrm{MP}=$ range of motion at the metacarpal-phalangeal joint

$\mathrm{PIP}=$ range of motion at the proximal interphalangeal joint

DIP $=$ range of motion at the distal interphalangeal joint

Table 2. Evaluation Scheme of Total Active Motion System (TAM).

\begin{tabular}{|c|c|}
\hline Grade of MOTION ACHIEVED & FINAL $\%$ of NORMAL TAM ACHIEVED \\
\hline EXCELLENT & No difference between TAM and normal \\
\hline GOOD & TAM $>75 \%$ of normal \\
\hline FAIR & TAM $>50 \%$ of normal \\
\hline POOR & TAM $<50 \%$ of normal \\
\hline
\end{tabular}


These included "surgical site infections" as defined by the Centers for Disease Control [17], wound edge necrosis, cellulitis at the surgical site, need for surgical intervention secondary to surgical site complications for any reason, need for administration of antibiotics for surgical site complication, prolonged or excessive draining, or prolonged healing of the surgical wound.

The amount of cigarettes smoked on a daily basis were also recorded, in order to draw conclusions on the existence of a dose dependent relationship and the outcome measures. Smokers classified as "Light" (less than 15 cigarettes per day) or "Heavy" smokers (more than or equal to 15 cigarettes per day), as devised by the world health organization and used by Justan.

Patient sex and distribution of tendon injuries were analyzed via a Chi-Square Test $(p<0.05$ level of significance, with $95 \%$ Confidence Interval "CI"). ROM was analyzed with an T-Test analysis ( $p<0.05$, with $95 \%$ CI). A Cohen's D Effect Size analysis was used to evaluate the relative effect on ROM in smokers vs non-smokers. T-test and Chi Squared test were used to evaluate the differences between "light" and "heavy" smokers".

\section{Results}

Analysis between the 2 groups (smoker vs non-smokers) revealed no significant difference between distribution of age, sex ( $p=$ $0.677,95 \% \mathrm{CI}$ ), proportions of flexors vs. extensor cases ( $\mathrm{p}$ $=0.809,95 \% \mathrm{CI})$ or injured thumbs $(\mathrm{p}=0.382,95 \% \mathrm{CI})$ as compared to the lesser digits. Surgical wound complication numbers were exceptionally low; 1 complication in the smokers (operative site infection successfully treated with oral antibiotics), and 2 complications in the non-smokers (1 case of Reflex Sympathetic Dystrophy and 1 case of excessive scar tissue warranting tenolysis).

Percentage of TAM regained in smokers was $70 \%$ of normal vs $75 \%$ TAM regained by non-smokers, with no significant difference between the groups $(p=0.406,95 \% \mathrm{CI})$. TAM grading of excellent, good, fair, and poor between smokers and nonsmokers displayed no significant differences in the proportion within each group ( $p=0.357,95 \% \mathrm{CI}$ ). When grouping all excellent and good results, vs the combination of fair and poor, there was not a significant difference $(p=0.936,95 \% \mathrm{CI})$. When grouping all excellent, good and fair results, vs poor, there still was no significant difference ( $p=0.253,95 \% \mathrm{CI}$ ). This tells us that tendon repairs universally do "poor" in terms of total active ROM, both flexor or tendon repairs, smoker or non-smoker.

Extensors tendon repairs fared significantly better with higher $\mathrm{ROM}$ in both the smoking and non-smoking groups vs flexors ( $\mathrm{p}$ $=0.028$ and $\mathrm{p}=0.037$ respectively). Smokers displayed a larger difference in the average ROM between flexors and extensors. Essentially, smoking had a greater influence on flexor tendon ROM than on extensors. This can be quantified using Cohen's D Effect Size, which was 0.532 (correlation coefficient $=0.257$ ) for flexor tendons (indicative of a medium effect), vs -0.086 (correlation coefficient $=-0.043$ ) for extensor tendons (indicative of a small effect). This is demonstrated in Figure 1, in the bell distributions of the percentage TAM regained in smokers and non-smokers for flexors and extensors is displayed. The closer overlap of the 2 bell shaped curves of the extensor tendons displays a close relation between smokers and non-smokers. This is in contrast to the lesser degree of overlap between the smokers and non-smokers of flexor injuries. This displays a larger effect of smoking on flexor tendons as compared to extensor tendons, although not to a clinically significant level.

No statistically significant difference exists between flexor tendon injury smokers vsnon-smokers, in terms of percentage of TAM regained $(p=0.191,95 \% \mathrm{CI}$ ). No statistically significant difference exists between extensor tendon injury smokers vsnon-smokers, in terms of percentage of TAM regained $(\mathrm{p}=0.852,95 \% \mathrm{CI})$.

Comparing "light" vs "heavy" smokers, there is no significant dose dependent effect in regards to numerous parameters; percent TAM regained for flexors and extensors combined $(\mathrm{p}=$ $0.762)$, percent TAM for flexors $(p=0.422)$, or percent TAM for extensors $(p=0.699)$. There was no significant difference between light and heavy smokers for excellent combined with good and fair results, vs. poor results in ROM for flexors and extensors combined $(p=0.697$ ) or excellent combined with good, vs. fair combined poor results for ROM in flexors and extensors combined $(p=0.436)$.

\section{Discussion}

There are only 2 noteworthy published papers displaying the effects of smoking on tendon injury, that of Justan and Trumble,

Figure 1. Distribution of the Percentage Total Active Motion Regained in Smokers and non Smokers for Flexors and Extensors.

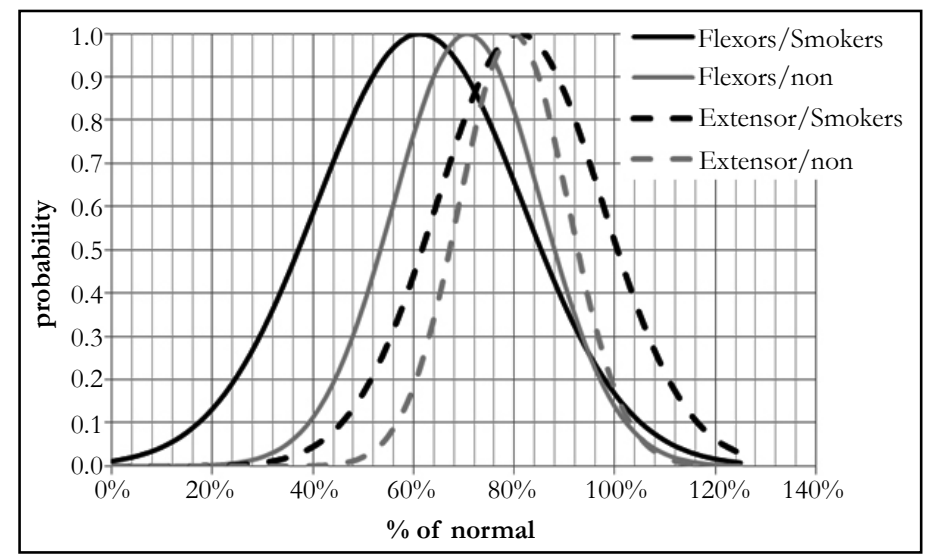


both of which different significantly from this paper in multiple aspects. The methodology of previous papers created numerous confounding variables which skewed their data. Trumble described the use of 16 surgeons in 8 surgery centers, vs Justan who claimed, "58 autologous tendon grafts harvested ... All the patients were operated on at our department by different surgeons". Trumble claims "all had received a Certificate of Added Qualification in hand surgery", whereas Justan does not make any comment on the level experience in hand surgery for the numerous surgeons involved in his study. There was 1 fellowship trained orthopedic hand surgeon used in this study, thus eliminating confounding variables in terms of surgical technique or materials used. The retrospective nature of this study could be considered a drawback. However, the consistency of the treatment provided and the rigid inclusion and exclusion criteria offset the retrospective nature of the study.

Prior reports ignore acute repair within 3 weeks from injury, a common practice. Reporting on tendon injury outcomes without making note of the time between injury and repair is not an accurate means to compare data, as it is well known prolonged immobilization of structures in the hand can lead to varying degrees of soft tissue contracture and decreased ROM. Previous studies do not signify the time frame their patients incurred the injury vs date of repair, a significant confounding variable in data analysis. Patients in this study were all treated within 3 weeks of the traumatic injury, a common scenario seen in most clinics.

This is the only paper to evaluate post-operative surgical site complications in smokers for acutely treated hand tendon injuries. The findings were surprising, in that of the 56 injured digits, only $3(\sim 5.4 \%)$ experienced complications, 2 of which were in the nonsmoking cohort. Therefore, this study cannot claim smoking has a significant effect on post-operative wound complications. Further research in this field is warranted and perhaps a larger sample population may reveal more information. The study shows that the effects on ROM status-post tendon injury repair, are more common than infection or post-operative complications.

The authors of this paper used a TAM grading system as set forth by the ASSH, widely used by occupational therapists and hand surgeons. This measurement takes into consideration ROM at the MP, PIP and DIP joints. This global ROM evaluation provides an accurate assessment of overall functional capacity of the digit in question, rather than solely measuring PIP and DIP ROM as done by Justan and Trumble. In terms of patient function, all corresponding joints (MP, PIP, and DIP) are used in combination in the real world attempting to utilize a digit. Therefore, it is misleading to only analyze a segment of that ROM and proclaim differences in smokers vs non-smokers as clinically significant. Justan claimed the Strickland method (measuring PIP and DIP) was a more accurate assessment in his paper as compared to TAM, and Trumble solely focused on PIP and DIP motion.

Trumble states; "Patients who smoked had less mean combined flexion $(130 \pm 9$ compared with $146 \pm 18) \ldots$ than did patients who did not smoke...." Although, he goes on to state; "We could not identify a significant difference between the smokers and nonsmokers with regard to DASH scores, the Jebsen-Taylor and Purdue pegboard dexterity test scores, or patient satisfaction scores." This statement correlates to our findings of TAM grading system with excellent, good, fair and poor, in that there is no appreciable clinical/functional difference noted between smokers and non-smokers in terms of ROM, but that these injuries are overall deleterious in terms of final ROM to the patient regardless of the smoking status. Grouping TAM in terms of grades rather than percentage regained, provides a more accurate correlate to functional capability. For example, data may show a statically significant presence of patients in one group with 10 degrees greater ROM, but clinically the functional repercussions may be negligible.

Individuals have investigated flexor tendons and the effect of smoking in the past, in respect to zone 2 flexor injuries in the Trumble paper and flexor tendon grafting as described by Justan. This is the first paper to evaluate the effects of smoking on ROM or wound complications on extensor tendon injuries. There is no statistically significant difference in terms of ROM, when taking percentage of TAM regained and the grading method into account, thus correlating to no gross functional effects attributed to smoking for extensor tendon injury repairs in this study.

There is no significant correlation between "Light" (less than 15 cigarettes per day) or "Heavy" smokers (more than or equal to 15 cigarettes per day) in terms of ROM as found in this study, in agreement with Justan. Thus, there is no significant dose dependent effect of smoking on ROM. Although, it appears flexors are more affected by smoking vs extensors (Figure 1). This is likely secondary to the relative complex anatomy of flexor zone 2 , making the effects of smoking manifest to a greater degree than in extensors. The anatomy of the extensor tendons is not as complex as flexor tendons, therefore adhesions around the tendinous structures may not have as great an effect on gross function as flexor tendons. In addition, the synergistic nature of the unique inter-tendinous connections of the digit extensors (juncture tendinae), likely aids in the rehabilitation process to increase early post-operative motion, therefore limiting adhesions commonly seen with immobilization. Therefore, there is some measureable effect of smoking on ROM, but not of statistical or clinical significance between flexor smokers and non-smokers. If smoking had no effect on tendon repairs, than the 2 bell shaped curves would look identical and the Cohen D Effect would be equal, this is obviously not the case.

The musculoskeletal manifestations of smoking correlate to effects on a cellular level. Nicotine imparts direct damage to $\mathrm{RBC}$ precursors, macrophages, and fibroblasts, creates a vasoconstrictive, hypercoagulable state with thrombogenic effects, and inhibits of revascularization [18]. These collectively limit oxygen and nutrient delivery to tissues, in effect retarding the process healing. Disruption of the actions of macrophages leads to prolonged healing, in that necrotic and waste materials and invading microbes are unable to be efficiently cleared from the wound. This combined with the relatively hypoxic environment of wounds in cigarette smokers, leads to prolonged and tenuous healing [19-22]. Smoking therefore reduces blood flow in tissues, impairing and prolonging the healing process. Based on the findings of this study, we propose the innate rich vascular supply to the hand accommodates in large part for these deleterious effects seen to a relatively greater extent elsewhere in the body. If these effects were seen to the same degree in the hand, the findings of our study would should greater statistical differences in our endpoints of ROM and surgical complications in smokers vs non-smokers. 
The effects on fibroblasts and myofibroblasts have been investigated in regards to smoking [23]. Smokers have approximately 1.8 times less mature collagen in their surgical wounds than non-smokers [24]. The prolonged healing time secondary to the cellular effects of smoking, likely leads to increased amounts of this immature collagen deposition [25, 26]. We propose that although the collagen seen in smokers is not mature and therefore lacks the biomechanical strength it would otherwise have, the increased deposition of this material secondary to the prolonged healing process likely leads to increased net fibrosis, translating to decreased ROM.

Smoking has been linked to numerous pathological states which are attributed to decreased health and premature death. Despite the undisputable harsh effects of smoking, it is scientifically inaccurate to claim patient post-operative ROM in flexor and extensor tendon injuries is significantly affected by this unhealthy habit. Nor can we claim surgical site complications are more common in smokers in the setting of such injuries. We call for increased research in this field to add to the medical knowledge, to better assess the factors which effect patient outcome and therefore better target those issues in order to provide superior care to our patients. By deriving what is truly deleterious to patient outcome, better outcomes and greater patient satisfaction can be achieved.

\section{References}

[1]. SR McClave A, James C, Caraballo R, Kaufmann R, Pechacek T (2010) Vital signs: current cigarette smoking among adults aged $\geq 18$ years—United States, 2009. Morbidity and Mortality Weekly Report (MMWR). 59(35): $1135-1140$.

[2]. Bodin J, Ha C, Sérazin C, Goldberg M, Leclerc A, et al., (2012) Effects of individual and work-related factors on incidence of shoulder pain in a large working population. J Occup Health. 54(4): 278-288.

[3]. Carbone S, Gumina S, Arceri V, Campagna V, Fagnani C, et al., (2012) The impact of preoperative smoking habit on rotator cuff tear: cigarette smoking influences rotator cuff tear sizes. J Shoulder Elbow Surg. 21(1): 56-60.

[4]. Safran MR, Graham SM (2002) Distal biceps tendon ruptures: incidence, demographics, and the effect of smoking. ClinOrthopRelat Res. (404): 275283.

[5]. Schneider A, Bennett JM, O'connor DP, Mehlhoff T, Bennett JB (2009) Bilateral ruptures of the distal biceps brachii tendon. J Shoulder Elbow Surg. 18(5): 804-807.

[6]. Galatz LM, Silva MJ, Rothermich SY, Zaegel MA, Havlioglu N, et al., (2006) Nicotine delays tendon-to-bone healing in a rat shoulder model. J
Bone Joint Surg Am. 88(9): 2027-2034.

[7]. Song EK, Rowe SM, Chung JY, Moon ES, Lee KB (2004) Failure of osteointegration of hamstring tendon autograft after anterior cruciate ligament reconstruction. Arthroscopy. 20(4): 424-428.

[8]. Sorensen LT, Karlsmark T, Gottrup F (2003) Abstinence from smoking reduces incisional wound infection: a randomized controlled trial. Ann Surg. 238(1):1-5.

[9]. Reichman DE, Greenberg JA (2009) Reducing surgical site infections: a review. Rev Obstet Gynecol. 2(4): 212-221.

[10]. Hoogendoorn JM, Simmermacher RK, Schellekens PP, Van der werken C (2002) Adverse effects of smoking on healing of bones and soft tissues. Unfallchirurg. 105(1): 76-81.

[11]. Castillo RC, Bosse MJ, MacKenzie EJ, Patterson BM, LEAP Study Group (2005) Impact of smoking on fracture healing and risk of complications in limb-threatening open tibia fractures. J Orthop Trauma. 19(3): 151-157.

[12]. Justan I, Ovesná P, Kubek T, Hýža P, Stupka I, et al., (2011) The effect of smoking on post-operative finger range of motion in patients with tendon grafts. In Vivo. 25(4): 697-702.

[13]. Trumble TE, Vedder NB, Seiler JG, Hanel DP, Diao E, et al., (2010) ZoneII flexor tendon repair: a randomized prospective trial of active place-andhold therapy compared with passive motion therapy. J Bone Joint Surg Am. 92(6): 1381-1389.

[14]. American Society of Hand Therapists (1992) Clinical Assessment Recommendations. 2nd (Edn), $401 \mathrm{~N}$ Michigan Ave, Chicago IL, 138.

[15]. ASSH (1990) The Hand, Examination and Diagnosis. American Society for Surgery of the Hand. 3rd (edn), Churchill Livingstone New York, 60.

[16]. Hunter, Mackin, Callahan (1995) Rehabilitation of the Hand: Surgery and Therapy. 4th (edn), Mosby. 1859-1864.

[17]. http://www.cdc.gov/hicpac/SSI/table1-SSI.html. February 24, 2015.

[18]. Wong LS, Martins-Green M (2004) First hand cigarette smoke alters fibroblast migration and survival: implications for impaired healing. Wound Repair Regen. 12(4): 471-484.

[19]. Jensen JA, Goodson WH, Hopf HW, Hunt TK (1991) cigarette smoking decreases tissue oxygen - Arch Surg1991. 126(9): 1131-1134.

[20]. Jones JK, Triplett RG (1992) The relationship of cigarette smoking to impaired intraoral wound healing: a review of evidence and implications for patient care. J Oral Maxillofac Surg. 50(3): 237-239.

[21]. Karim A, Pandit H, Murray J, Wandless F, Thomas NP (2006) Smoking and reconstruction of the anteriorcruciate ligament. j bone joint surg. 88(8): 1027-1031.

[22]. Kwiatkowski TC, Hanley EN, Ramp WK (1996) Cigarettes smoking and its orthopedics consequences. Am J Orthop. 25(9): 590-597.

[23]. Duygulu F, Karaoglu S, Zeybek ND, Kaymaz FF, Gunes T (2006) The effect of subcutaneously injected nicotine on achilles tendon healing in rabbits. Knee Surg Sports Traumatol Arthrosc. 14(8): 756-761.

[24]. Alicioglu B, Ulucam E, Cigali BS, Yilmaz A, Taskinalp O (2009) The effect of smoking on tendon thickness and degeneration in young amateur sportspeople. Dicle Med J. 36(1): 16-22.

[25]. Clark RF (1996) The Molecular and Cellular Biology of Wound Repair. New York, Plenum Press, Springer - US.

[26]. Wong LS, Green HM, Feugate JE, Yadav M, Nothnagel EA, et al., (2004) Effects of "second-hand" smoke on structure and function of fibroblasts, cells that are critical for tissue repair and remodeling. BMC Cell Biol. 5(1): 13. 